\title{
Abbreviations - General
}

abbrev.

abs.

adj.

adv.

Af.

Akk.

AnatArab.

Arab.

Ass.

BK

Class.

comp.

conj.

сp.

crit. app.

cstr.

der.

det.

Eng.

ES

Eshtaf.

Ethpa.

Ethpe.

Ettaf.

Europ.

f.

fig.

FK

fut.

Germ.

GN

imp. abbreviation; abbreviated

status absolutus, absolute state

adjective; adjective phrase

adverb; adverb phrase

Af'el

Akkadian

Anatolian Arabic

Arabic

Assyrian

Behiyye Korkunc, wife of FK

Classical Syriac

compound, component

conjunction; conjunction phrase

compare

critical apparatus

status constructus, construct state

derived; derivative(s)

status determinatus, determined state

English

East Syriac

Eshtaf'al

Ethpa'al

Ethpe'el

Ettaf ‘al

European languages

feminine

figurative

Faulus Korkunc, our main informant for

Turoyo

future tense

German

geographical name

imperative 
impf.

inf.

intr.

Ital.

Kurd.

$\mathrm{m}$.

MLS

MPers.

n.

NPers.

NT

obj.

OPers.

OT

$\mathrm{Pa}$.

Pe.

pf.

pl.

PN

pol.

prep.

pres.

pron.

pseudo-inf.

ptc. a.

ptc. p.

$Q$

quad.

R

RT

sg. imperfect

infinitive

intransitive

Italian

Kurdish

masculine

Modern Literary Syriac

Middle Persian

noun, substantive; noun phrase

New Persian

New Testament

object

Old Persian

Old Testament

Pacel

Pe'al

perfect

plural

personal name

political, politically

preposition, prepositional phrase

present tense

status pronominalis, pronominal state; also pronominal

pseudo-infinitive

participle active

participle passive

anonymous informant from Qamishli (Syria)

quadriliteral root or conjugation

Turoyo quoted from texts published by Hellmut Ritter (often RT).

Rural (spoken) Turoyo (as distinct from WT), often from FK.

singular 
Shaf.

suff.

Swed.

syn.

t-quad.

tr.

Tu.

Turk.

var.

vb.

vb. n.

WS

WT
Shaf'el

suffix

Swedish

synonym(s); synonymous

quadriliteral root or conjugation with t- infix. transitive

Turoyo, without distinction between RT and WT

\section{Turkish}

variant

verb (phrase), verbal

verbal noun

West Syriac

Written Turoyo (as distinct from RT). WT indicates forms found either in dictionaries, word lists or in written texts. 
